\title{
Kosten und Finanzierung des Gesundheitswesens 2007
}

Gerhard Kocher

Siehe zum Thema

Gesundheitskosten auch das «Zu guter Letzt» von Anna Sax.
Korrespondenz:

Dr. rer. pol. Gerhard Kocher

Haldenweg $10 \mathrm{~A}$

$\mathrm{CH}-3074$ Muri

gerhard.kocher@muri-be.ch

\section{Die Kostenblöcke und ihre Entwicklung seit 2002}

Das Bundesamt für Statistik hat im Dezember die Zahlen für das Jahr 2007 veröffentlicht [1]. Die Gesamtkosten des Gesundheitswesens belaufen sich auf 55,2 Mrd. Franken (Tabelle 1). Die stationäre Behandlung kostete 25,2 Mrd. die ambulante Behandlung inklusive Zahnmedizin 17,4 Mrd. Franken.

Von den übrigen Ausgaben fallen besonders die Medikamente mit 5,7 Mrd. Franken ins Gewicht. Dabei ist zu beachten, dass die wahren Ausgaben etwa 1,3 Mrd. Franken höher sind: Die Medikamente der Spitäler in etwa dieser Höhe sind nicht in den 5,7 Mrd. Franken enthalten, sondern aus schwerverständlichen Gründen - und nur teilweise - unter der Rubrik stationäre Behandlung. Diese 7 Mrd. Franken entsprechen $12,6 \%$ der nationalen Gesundheitsausgaben und sind damit erheblich höher (22\%) als die vor allem von der Pharmaindustrie oft genannten rund 10,3\%.

Zwischen 2002 und 2007 stiegen die Pro-KopfGesundheitsausgaben von 6454 auf 7247 Franken pro Jahr, was einer Zunahme von 12,3\% entspricht. Die weitaus grösste prozentuale Kostensteigerung war bei den ambulanten Spitalleistungen zu verzeichnen $(+36 \%)$. Diese Entwicklung ist allerdings zu einem grossen Teil beabsichtigt und erwünscht. Sie trägt dazu bei, dass die stationären Behandlungskosten der Krankenhäuser zwar der weitaus grösste, aber prozentual am schwächsten wachsende Kostensektor sind (+5,2\%).

Die beträchtlichen Zunahmen der Ausgaben für Spitex (19,5\%) und Prävention (17,5\%) sind im Zug einer Neuorientierung des Gesundheitswesens eben-

Abbildung 1

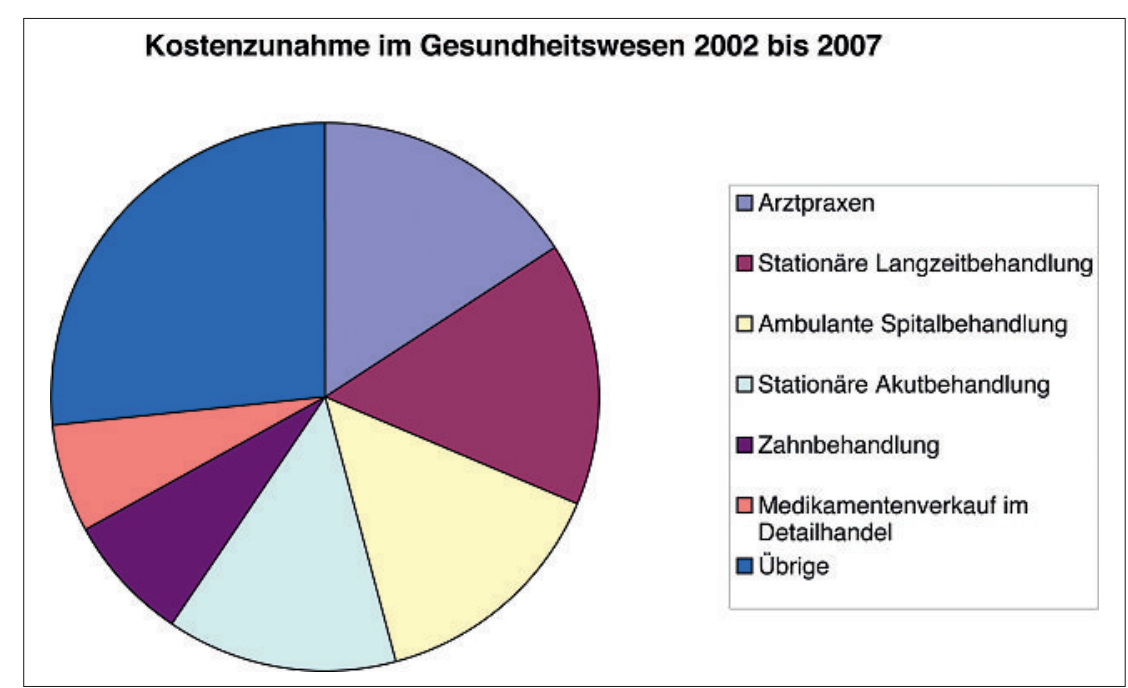

\section{Coûts et financement du système} de santé en 2007

L'Office fédéral de la statistique a publié les chiffres détaillés des coûts de la santé en 2007. Le total des coûts s'élève à 55,2 milliards de francs. Les traitements hospitaliers reviennent à 25,2 milliards, les traitements ambulatoires, médecine dentaire inclus, à 17,4 milliards. Par rapport aux autres dépenses, une part importante est à imputer aux médicaments en particulier, avec 5,7 milliards de francs (plus exactement 7 milliards).

Entre 2002 et 2007, les dépenses de santé par habitant ont augmenté de $12,3 \%$ et sont passées de Fr. 6454.- à Fr. 7247.- par an. La plus forte augmentation exprimée en pourcentage revient aux prestations hospitalières ambulatoires $(+36 \%)$. Toutefois, cette progression est en grande partie intentionnelle et souhaitée.

Une comparaison de l'augmentation des coûts en francs entre 2002 et 2007 permet d'identifier la plus grande augmentation chez les médecins (cabinets médicaux) qui ont coûté 1,2 milliard de plus qu'en 2002. Suivent les traitements hospitaliers de longue durée avec 1,1 milliard ainsi que les soins hospitaliers aigus et les traitements hospitaliers ambulatoires avec 1 milliard d'augmentation chacun.

Le rapport de I'OFS présente le financement des dépenses de santé du point de vue de la sûreté sociale: ménages/particuliers (65,8\%), Etat (16,2\%), entreprises $(9,4 \%)$, assurances sociales $(7,6 \%)$ et autres sources privées (1\%).

falls eher positiv zu bewerten. Dazu kommt, dass diese beiden Ausgabengruppen zusammen nicht einmal 5\% (2007) der Gesamtausgaben ausmachen.

Vergleicht man den Kostenzuwachs 2002 bis 2007 statt prozentual in Franken (Abb. 1), zeigt sich bei den Ärzten (Arztpraxen) die grösste Kostensteigerung: 1,2 Mrd. Franken mehr als im Jahr 2002. Es folgen die stationäre Langzeitbehandlung mit 1,1 Mrd. und die stationäre Akutbehandlung sowie die ambulante 
Spitalbehandlung mit je 1 Mrd. Franken Mehrkosten. Diese vier Bereiche machen $60 \%$ der Kostenzunahme 2002 bis 2007 aus. Erhebliche Mehrausgaben sind auch bei den Zahnärzten und beim Medikamentenverkauf im Detailhandel zu verzeichnen. Zu berücksichtigen ist, dass die Bevölkerung in dieser Zeitspanne jährlich um $0,8 \%$ wuchs (2008 um 108000 Personen).

Hochinteressant ist eine weitere BFS-Statistik im Internet [2]: Die Gesundheitskosten 2007 nach Alter und Geschlecht und aufgeschlüsselt nach Leistungen.

Tabelle 1

Kosten nach Leistungen 2007

\begin{tabular}{|c|c|c|}
\hline & Mio Fr. & $\%$ \\
\hline Stationäre Behandlung: & 25173,3 & 45,6 \\
\hline Akutbehandlung inkl. Psychiatrie & 14793,1 & 26,8 \\
\hline Rehabilitation & 863,3 & 1,6 \\
\hline Langzeit & 7191,4 & 13 \\
\hline Andere (1) & 2325,5 & 4,2 \\
\hline Ambulante Behandlung: & 17405,9 & 31,5 \\
\hline Ärzte & 7856,3 & 14,2 \\
\hline Krankenhäuser & 3710,4 & 6,7 \\
\hline Zahnbehandlung & 3550,7 & 6,4 \\
\hline Physiotherapie & 705,8 & 1,3 \\
\hline Psychotherapie & 190,3 & 0,3 \\
\hline Spitex & 1212,4 & 2,2 \\
\hline Andere paramedizinische Leistungen & 180 & 0,3 \\
\hline Andere Leistungen: & 1831,3 & 3,3 \\
\hline Medizinische Laboruntersuchungen (2) & 842,1 & 1,5 \\
\hline Radiologie (3) & 162,3 & 0,3 \\
\hline Transport und Rettung & 827 & 1,5 \\
\hline Andere & 0 & 0 \\
\hline Verkauf von Gesundheitsgütern: & 6761,1 & 12,2 \\
\hline Arzneimittel durch Detailhandel & 3956,3 & 7,2 \\
\hline Arzneimittel durch Ärzte & 1733,6 & 3,1 \\
\hline Therapeutische Apparate & 1071,2 & 1,9 \\
\hline Prävention: & 1281,2 & 2,3 \\
\hline Alkohol- und Drogenmissbrauch & 185,5 & 0,3 \\
\hline Infektionskrankheiten & 169,6 & 0,3 \\
\hline Lebensmittelkontrolle & 132,5 & 0,2 \\
\hline Schulgesundheit & 180,5 & 0,3 \\
\hline Gesundheitsförderung (Art. 19 KVG) & 18,7 & 0 \\
\hline Berufskrankheiten und Unfälle (4) & 123,4 & 0,2 \\
\hline Andere (5) & 470,9 & 0,9 \\
\hline Verwaltung: & 2762,1 & 5 \\
\hline Öffentliches Gesundheitswesen & 523,7 & 0,9 \\
\hline Krankenversicherung KVG & 987,1 & 1,8 \\
\hline Unfallversicherung UVG & 149,3 & 0,3 \\
\hline IV-AHV & 227,9 & 0,4 \\
\hline Private Krankenversicherung VVG & 874,2 & 1,6 \\
\hline Total & 55214,9 & 100 \\
\hline
\end{tabular}

(1) Institutionen für Behinderte, Suchtkranke und für Personen mit psychosozialen Problemen.

(2) In medizinischen Labors, ohne Krankenhäuser und Arztpraxen.

(3) Röntgeninstitute und Spezialärzte für Radiologie, ohne Krankenhäuser und andere Arztpraxen.

(4) Verhütung von Unfällen, Berufskrankheiten und Nichtberufsunfällen, Leistungen der UVG

(5) Leistungen der Gesundheitsorganisationen (ohne Erwerbscharakter).
Die 6-10-Jährigen kosten jährlich 1666 Franken, die 96-Jährigen und Älteren 70160 Franken (es sind aber nur 1026 Personen).

\section{Die Finanzierung der nationalen Gesundheitsausgaben}

Der BFS-Bericht zeigt die Finanzierung der 55,2 Mrd. Franken Gesundheitsausgaben aus drei Sichtwinkeln:

- Finanzierung nach Direktzahlenden: Wer zahlt den Leistungserbringern ihre Leistungen? $\mathrm{Zu} \mathrm{42,9 \%}$ sind es die Sozialversicherungen, zu 30,7\% die privaten Haushalte, zu 16,2\% der Staat, zu 9,2\% die Privatversicherungen, und $1 \%$ wird anders, privat, finanziert.

- Finanzierung aus der Perspektive der sozialen Sicherheit: Hier werden auch die Transferzahlungen ausgewiesen: Prämienverbilligung, Sozialhilfe, Ergänzungsleistungen und Hilflosenentschädigung. Die Gesamtfinanzierung erfolgt hier durch private Haushalte $(65,8 \%)$, den Staat $(16,2 \%)$, Unternehmen $(9,4 \%)$, Sozialversicherungen $(7,6 \%)$ und durch andere private Quellen (1\%). Im internationalen Vergleich sind unsere Out-of-pocket-Zahlungen ausserordentlich hoch. Diese Direktzahlungen der Patienten an Leistungserbringer für nicht versicherte Leistungen wie z. B. 91\% der Zahnmedizin und für einen Teil der Medikamente betragen rund 12 Mrd. Franken

- Finanzierung aus volkswirtschaftlicher Sicht: Hier wird gezeigt, wer letztendlich die finanzielle Last des Gesundheitswesens trägt: die privaten Haushalte $(66,8 \%)$, der Staat $(26,8 \%)$ und die Unternehmen mit $6,4 \%$

Die BFS-Broschüre erscheint jeweils erst fast zwei Jahre nach Ablauf des Berichtsjahres. In der Zwischenzeit sind teilweise schon aktuellere Zahlen (für 2008) publiziert worden [3]. Die BFS-Broschüre ist aber der einzige detaillierte Überblick mit gesicherten Zahlen und ist deshalb von grossem Wert. Ein Ausblick: Für 2009 prognostiziert die KOF Konjunkturforschungsstelle der ETH die Gesamtausgaben auf 60,7 Mrd. Franken (höher als das Jahresbudget des Bundes). Dies wäre eine $\mathrm{Zu}$ nahme von nicht weniger als 2,5 Mrd. Franken oder $4,3 \%$ gegenüber 2008 .

\section{Literatur}

1 Kosten und Finanzierung des Gesundheitswesens 2007 - Coûts et financement du système de santé en 2007. Zweisprachig, Neuchâtel 2009, 50 Seiten. Kostenlos als pdf-Datei unter: www.bfs.admin.ch/bfs/ portal/de/index/themen/14/22/publ.Document.127959.pdf. Der Bericht wird auch als Broschüre erscheinen.

2 www.statistik.admin.ch $\rightarrow$ Infothek $\rightarrow$ Statistisches Lexikon $\rightarrow$ Zugang zum Lexikon $\rightarrow$ Themen: 14 Gesundheit $\rightarrow 14.5$ Kosten, Finanzierung

3 Beispiele: Krankenhausstatistik 2008 - Standardtabellen sowie Statistik der sozialmedizinischen Institutionen 2008 - Standardtabellen. Beide BFS, Neuenburg 2009, online und als Broschüren erhältlich. 